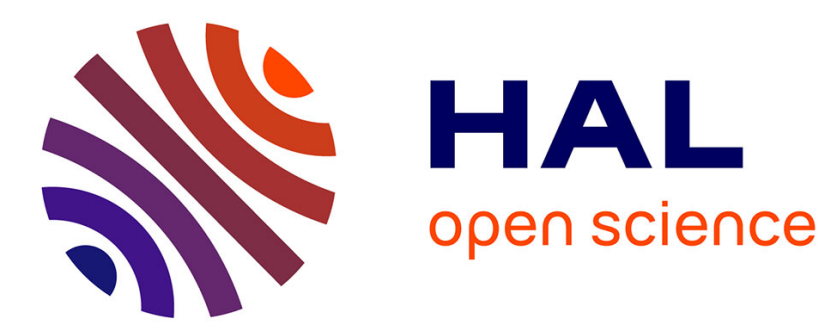

\title{
2-D evanescent trapping of colloids in the vicinity of a micrometer waveguide
}

O Emile, Janine Emile, Herve Tabuteau

\section{To cite this version:}

O Emile, Janine Emile, Herve Tabuteau. 2-D evanescent trapping of colloids in the vicinity of a micrometer waveguide. EPL - Europhysics Letters, 2017, 116 (6), pp.64003. 10.1209/02955075/116/64003. hal-01467373

\section{HAL Id: hal-01467373 \\ https://hal.science/hal-01467373}

Submitted on 14 Feb 2017

HAL is a multi-disciplinary open access archive for the deposit and dissemination of scientific research documents, whether they are published or not. The documents may come from teaching and research institutions in France or abroad, or from public or private research centers.
L'archive ouverte pluridisciplinaire HAL, est destinée au dépôt et à la diffusion de documents scientifiques de niveau recherche, publiés ou non, émanant des établissements d'enseignement et de recherche français ou étrangers, des laboratoires publics ou privés. 


\title{
2-D evanescent trapping of colloids in the vicinity of a micrometer waveguide.
}

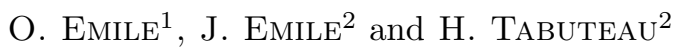 \\ 1 Université Rennes 1, 35042 Rennes cedex, France \\ 2 UMR CNRS 6251 IPR, Université Rennes 1, 35042 Rennes cedex, France
}

PACS 47.57. J- - Colloidal systems

PACS 42.50.Wk - Mechanical effects of light on material media, microstructures and particles

PACS 64.75.Xc - Phase separation and segregation in colloidal systems

\begin{abstract}
We report on the trapping of micrometer colloids using the evanescent wave from a multimode cylindrical optical waveguide. We show that the particle trapping is a two step process. With a low power visible laser injected in the device, particles are first captured at a radial distance twice greater than the diameter of the waveguide and then drawn near to it. In a second time particles turn around the waveguide and get trapped in a direction corresponding to the TM polarization of the laser. Such a device could be easily implemented in microfluidic systems in order to coat surfaces or to control particles deposition and assembly. Conversely, it could find applications in filtration process to aggregate and remove colloidal pollutants.
\end{abstract}

Introduction. - Since their first discovery $[1,2]$, optical tweezers have become an essential tool in physics, chemistry and biology to manipulate nano-to-micrometer objects $[3,4]$. However, because they are based on focused laser beams, conventional use of optical tweezers leads to a diffraction limited volume. New strategies based on evanescent waves and/or nanostructures substrates have been developed to reach the nanoscale trapping volume range [5-8]. In the near field, for example on the surface of a prism or under focused evanescent wave illumination, at total reflection $[9,10]$, the intensity gradient force of the evanescent wave could be steep, leading to a very efficient trapping. Curiously, in one or two dimension evanescent trapping, most of the experiments dealt with tapered fibers and submicron waveguides, in order to benefit from a deep penetrating evanescent wave around the guide [11-15]. However, although the intensity at the waveguide surface may be lowered, larger waveguides should in principle lead to reduced evanescent waves penetration and steeper intensity variations. It should then allow an efficient trapping at the surface of the guide. In this letter we address the trapping of $2 \mu \mathrm{m}$-diameter particles near a $3.5 \mu \mathrm{m}$-diameter post, using low power laser light. We report on the trapping mechanism and we show that it is a two step process.
Experimental set-up. - The wave guide is a PDMS (PolyDiMethilSiloxane) cylinder or post (diameter $d=$ $3.5 \pm 0.5 \mu \mathrm{m}$, height $h=15 \pm 5 \mu \mathrm{m}$ ) made on a glass slide by standard soft lithography [16]. We make array of posts $(10 \times 10$ square $)$ with a distance of $500 \mu \mathrm{m}$ between them. The precisions on the post dimensions are linked to the measurements performed with an optical microscope. Even if a single post is involved during an experiment, the data statistics results from the use of several posts. They are stuck to the upper side of the cell (see Fig.1). Particles flow in between the posts but also underneath them.

The dilute colloidal suspension $\left(10^{-4}\right.$ volume fraction) is made of $2 \mu \mathrm{m}$-diameter polystyrene particles (Life Tech.) dispersed in heavy water in order to increase the particles number that flow through the posts. We apply a pressure difference across the cell by using a pressure controller system (Elveflow OB1 Mk2) with an accuracy of $0.3 \mathrm{mbar}$, leading to a permanent flow. Particles are mostly advected, i.e. with a negligible Brownian motion. Depending on the applied pressure, the velocity of the trapped particles is between $8 \mu \mathrm{m} / \mathrm{s}$ and $20 \mu \mathrm{m} / \mathrm{s}$ corresponding to a Péclet number of the order of $10^{4}$. All the experiments are performed at $\mathrm{T}=20^{\circ} \mathrm{C}$.

The experimental set up is displayed on Fig. 1. A green frequency double YAG laser $(\lambda=532 \mathrm{~nm}$, Crystal laser, $\mathrm{P}=5 \mathrm{~mW})$ is focalized at the lower entrance of the post by a $\times 20$ objective (beam waist $1.5 \mu \mathrm{m}$ ). The post and 


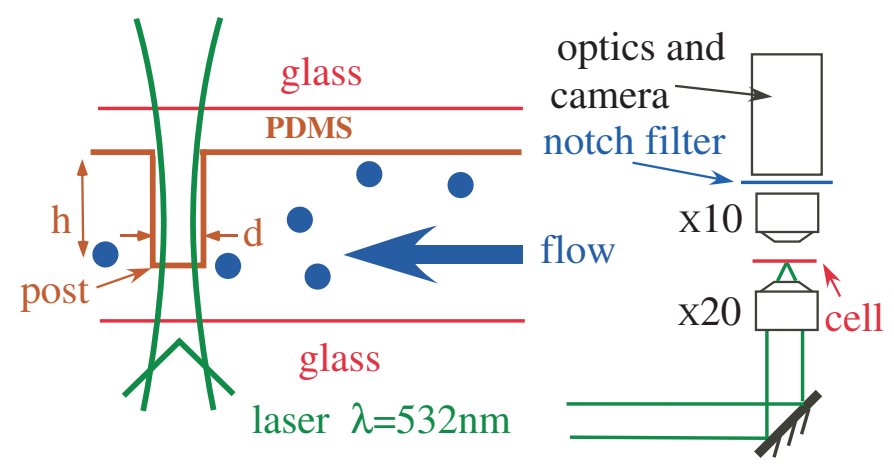

Fig. 1: Experimental set up. Drawing not to scale.

the particles are imaged with a $\times 10$ objective followed by an image expanding system and a camera (Edmund Scientific). The frame rate is 12.5 images per second. Images are analyzed using the free available ImageJ software. A Notch filter (Edmund Scientific $\mathrm{OD}=7$ ) is used to block most of the green light in order to be able to track the particles. In the green, at $\lambda=532 \mathrm{~nm}$, the optical index of the PDMS is $n_{2}=1.407$ [17], whereas the index of the dilute solution is close to the index of water $n_{1}=1.327$, and the index of the colloids is $n=1.596$. Since $n$ is higher than $n_{1}$ we get an efficient trapping of the particles by the optical gradient force $[1,2]$.

Results. - We have been able to track more than 400 particles as they flow in the vicinity of the post and eventually get trapped by the evanescent wave. A typical example of such a trajectory appears on Fig. 2. The first picture (top left corner of Fig. 2a) is the raw color picture. Although most of the green light has been blocked by the notch filter, it still saturates the camera. We have subtracted the green and the blue color images, keeping only the red one, in order to track the particles with a reasonable contrast.

We have then plotted the particle position versus time (see Fig. 2b). The trajectory can be divided in three different regimes. The particle first follows a fluid streamline that goes towards the post from $(t=0 \mathrm{~s}$ to $t=0.48 \mathrm{~s})$, at constant velocity $(17 \mu \mathrm{m} / \mathrm{s})$. This first regime is well resolved with our acquisition system, as can be seen on Fig. 2b. Similarly to the particle capture by a collector within a filter [18], one can define the distance between the particle and the post, $b$, called thereafter impact parameter.

In a second regime, the particle is captured by the evanescent wave near the post $(t=0.56 \mathrm{~s})$. Although the laser intensity is weak, this is a fast regime where the particle is suddenly attracted towards the post along a radial direction and then remains very close to it. We cannot temporally resolve this second regime with the acquisition rate we used. It is surely faster than $0.08 \mathrm{~s}$. We introduce a capture angle $\theta_{c}$ that corresponds to the angle for which the particle is attracted by the evanescent wave near the post. The zero angle corresponds to the horizontal axis on figure Fig. 2b.

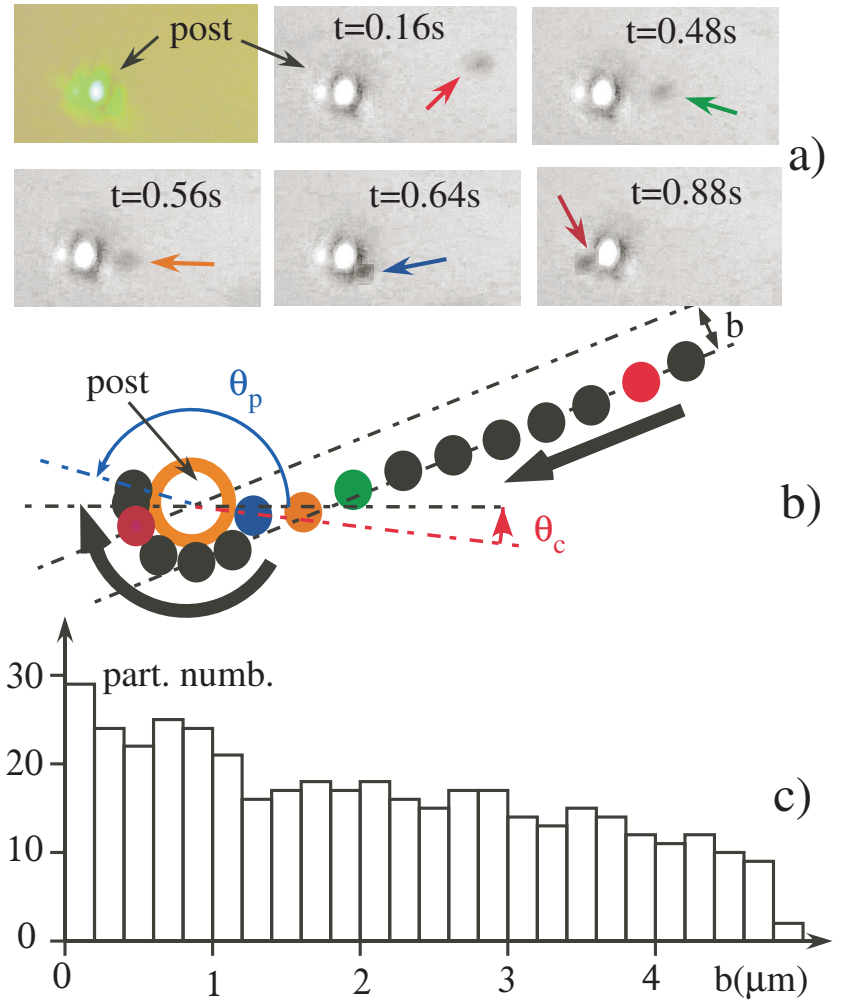

Fig. 2: a) Dynamics of a typical particle trajectory during the trapping process. The first image is a raw one while the other are corrected and correspond to the various times during the process. Arrow: position of the particle. b) Trajectory of the trapping process. The colored particles correspond to the colored arrows on the images. $\theta_{c}$ is the capture angle and $\theta_{p}$ the trapping angle. The zero angle corresponds to the horizontal direction. c) Number of particles captured for different values of the impact parameter $b$.

Finally, in a third regime, the particle rotates slowly around the post until it becomes still at a given position in a direction corresponding to an angle $\theta_{p}$ (from $t=0.64 \mathrm{~s}$ to $t=0.88 \mathrm{~s}$ ). This $0.2 \mathrm{~s}$ long-regime can be also resolved here as can be seen in Fig. 2b. Actually, the particle is not really at rest since it slightly oscillates around the trapping position. This is probably due to its residual Brownian motion of the particle. However it oscillates only in the angular direction, meaning that the restoring force is steeper in the radial direction than in the angular direction. It is worth noting that when the laser is switched off, the particle departs from the post following the flow direction. Therefore, the particle remains very close to the post only when the laser is hold on. Because of these three very different regimes, the trapping force must have a large intensity range. It thus could be used to probe forces within the suspension such as a Casimir force for example [19], using different sizes of particles.

From the analysis of the trajectories, one can investigate the statistics of the impact parameter for all the trapped particles. This is shown in Fig. 2b. The maximum impact 


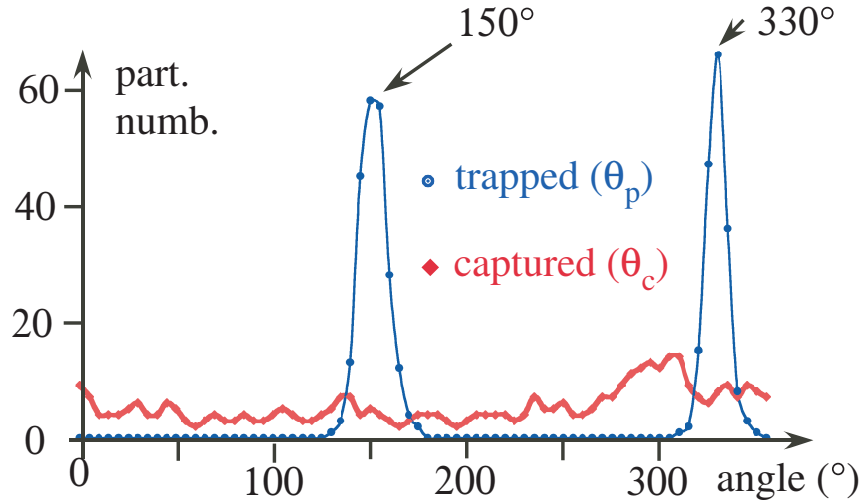

Fig. 3: Number of particles trapped versus capture angle $\theta_{c}$ (red curve), and versus trapping angle $\theta_{p}$ (blue curve). The step of the angles is $5^{\circ}$.

parameter for the particles to be trapped is $b_{\max }=5 \mu \mathrm{m}$. Thus, taking into account the size of the particles, the capture zone for the evanescent wave around the post is a $4.5 \mu \mathrm{m}$-radius cylindrical volume, leading to a capture range of the order of $2.25 \mu \mathrm{m}$. Here, the capture range is about twice the post diameter. However, it is well known that the penetration depth of the evanescent wave depends on the wavelength, the capture range should then depends also on the wavelength.

From Fig. $2 \mathrm{~b}$, the capture angle $\theta_{c}$ of this specific particle does not correspond to the trapping angle $\theta_{p}$. One may think that $\theta_{p}$ also depends on the flow, since here, $\theta_{p}$ is nearly opposed to the incident direction. Is this a general rule? We have registered the number of trapped particle for given angles $\theta_{c}$ and $\theta_{p}$ (see Fig. 3). First, the capture angle $\theta_{c}$ is rather isotropic, since we have changed the flow direction during the experiments (red curve). Yet, the particles are not staying where they are captured. They rotate around the post and stop at one of the two angles $\left(\theta_{p}=150^{\circ}\right.$ and $\left.\theta_{p}=330^{\circ}\right)$. These angles are symmetric regarded to the post and are independent of the flow direction. The next paragraph is devoted to the discussion of this observation.

\section{Discussion. -}

Evanescent modes. Since the diameter of the post is $3.5 \mu \mathrm{m}$ and the optical index difference between water and PDMS is 0.080 , the post is not a single-mode waveguide. One can get some insight of the light behavior and evaluate the number of modes propagating within the guide, using a simple one dimension model based on geometrical optics [20]. The optical rays are reflected on both sides and have to interfere constructively with the ray going straight (see Fig. 4a) [20]. This leads to the following equation

$$
2 k n_{2} d \cos \theta=2 \phi+2 \pi m
$$

where $k$ is the wave-vector modulus, $\theta$ the incidence angle, $\phi$ the phase shift experienced at total reflection, and $m$ the mode number. The phase shift is different for a TE or a
TM polarization $[22]$

$$
\begin{aligned}
\tan \left(\phi_{T E} / 2\right) & =-\left(n_{1} \beta\right) /\left(n_{2} \cos \theta\right) \\
\tan \left(\phi_{T M} / 2\right) & =-\left(n_{2} \beta\right) /\left(n_{1} \cos \theta\right)
\end{aligned}
$$

$\beta$ being equal to $\beta=\left[\left(n_{2} / n_{1} \sin \theta\right)^{2}-1\right]^{1 / 2}$. Here, since the two optical indexes are rather close to each other, the incidence angles are nearly the same. Nevertheless, Eq. 1 can be solved graphically for each polarization, as shown on Fig. 4b. Actually, the two curves of the left part of Eq. 1 superimpose. According to Fig. 4b, up to 6 modes can propagate. The fundamental mode corresponds to an incident angle which is far above the critical angle $\theta_{s}=\sin ^{-1}\left(n_{1} / n_{2}\right)$ whereas higher modes correspond to an incident angle closer to $\theta_{s}$. Besides, since the penetration depth $z_{d}$ equals $z_{d}=1 / k \beta$, the evanescent wave penetrates deeper for the higher modes than for the fundamental mode. The capture is performed by the highest mode, whereas all the modes participate to the trapping. However, since the $m=0$ has the lowest penetration depth (of the order of $0.3 \mu \mathrm{m}$ ), its intensity variation is the steepest, and it is thus the most efficient mode for trapping. For the $m=6$ mode we find a penetration depth of the order of $2 \mu \mathrm{m}$ in agreement with the capture range of $2.25 \mu \mathrm{m}$ found experimentally (see Fig. 2c).

The height of the post is about $h=15 \mu \mathrm{m}$. The particles are trapped in the upper zone of the post. The laser is injected from below. Then the modes propagating in the guide can be mixed so as the amplitude of the field is distributed among the various modes, although the fundamental mode has the highest amplitude.

We could have calculated the modes within the waveguide using a model of a cylindrical waveguide as it has been done in the literature (see for example [21]). However, there is an assumption that is usually done concerning the polarization of the field that is: the propagation is isotropic and then the field is treated as a scalar quantity. Nevertheless, we observe here an accumulation of the particles at specific angles. As explained in the next paragraph, this accumulation is due to an anisotropic total internal reflection of the field on the post surface. This induces a difference of the evanescent wave intensity between TE and TM polarizations.

Trapping angle. Let us now focus on the trapping angle $\theta_{p}$. The evanescent wave amplitude in the post vicinity equals to $t \times E_{0}, E_{0}$ being the amplitude of the incident wave and $t$ the modulus of the complex transmission coefficient given by [22]

$$
\begin{aligned}
t_{T E} & =\frac{2 n_{2} \cos \theta}{\sqrt{n_{2}^{2} \cos ^{2} \theta+n_{1}^{2} \beta^{2}}} \\
t_{T M} & =\frac{2 n_{2} \cos \theta}{\sqrt{n_{1}^{2} \cos ^{2} \theta+n_{2}^{2} \beta^{2}}}
\end{aligned}
$$

Such coefficients are plotted on Fig. 4c. One can note that $t_{T M}>t_{T E}$ as it is usually found with evanescent waves $[23,24]$. Precisely, in our case, the laser is linearly polarized with a polarization aligned with a direction corresponding to an angle of $150^{\circ}$. Thus $\theta_{p}=150^{\circ}$ 


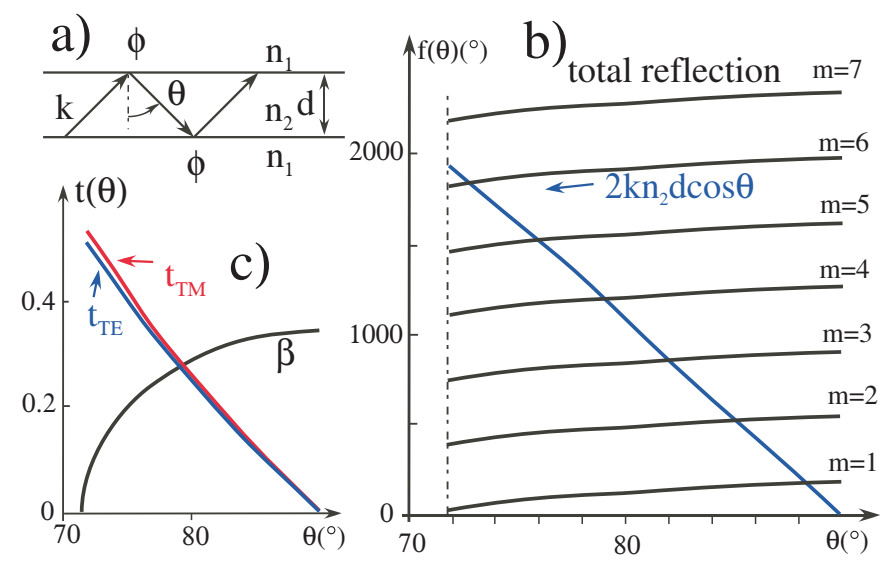

Fig. 4: a) Ray optics interpretation of the optical guiding. b) Graphical resolution of Eq. 1 for $d=3.5 \mu \mathrm{m}$. c) Transmission coefficients $t_{T E}$ and $t_{T M}$ of the evanescent wave for the two polarizations, and $\beta$ coefficient versus the angle of incidence.

corresponds to the TM polarization of the incident light. Around the post, there is a gradient of intensity which is maximal for $\theta_{p}$. It drives the particles towards this direction. This gradient in the azimuthal direction is smaller than the one due to the evanescent wave in the radial direction. In the capture zone, the particles are first attracted towards the surface of the post before rotating around it to the trapping angle, as observed experimentally (see Fig. $2 \mathrm{~b})$.

We have rotated the polarization of the laser with a polarization aligned with a direction equal to $120^{\circ}$ and $180^{\circ}$. We have observed that the particles are being trapped for a trapping angle $\theta_{p}=120^{\circ}$ and $\theta_{p}=180^{\circ}$ respectively. The particles are thus trapped in a direction that corresponds to the TM polarization of the laser beam.

Let us estimate the order of magnitude of the gradient forces. As can be seen on Fig. 2b, we can follow the rotation of the particle around the post. Using the fundamental principle of dynamics, the experimentally estimated force in the tangential direction is thus of the order of $F_{t} \approx \times 10^{-20} \mathrm{~N}$. Concerning the force in the radial direction, according to [25], the gradient force writes

$$
F_{\text {grad }}=\frac{2 n_{2} \pi r^{3}}{c}\left[\frac{m^{2}-1}{m^{2}+2}\right]\|\operatorname{grad}(I)\|
$$

where $r=1 \mu \mathrm{m}$ is the particle radius, $c$ is the celerity of light, $m=n_{2} / n_{1}$ and $I$ is the evanescent light intensity. In the radial direction the intensity gradient is equal to

$$
\|\operatorname{grad}(I)\|_{z}=\frac{I_{0}}{z_{d}} \exp \left(-z / z_{d}\right)
$$

where $I_{0}$ is the light intensity of the evanescent wave on the surface of the post. Assuming a waist of the $m=6$ mode equals to $4 \mu \mathrm{m}$, taking into account the expressions of Eq. 3 , given that around half of the power is injected in the guide, and that around $0.1 \%$ of the initial power ends the $m=6$ mode [26], one finds a force in the radial direction that is of the order of $F_{r} \approx \times 10^{-17} \mathrm{~N}$ at a distance of $1 \mu \mathrm{m}$ from the post. Closer to the post since the other modes are also playing a role, the force is much higher.

One can also calculate the intensity gradient in the tangential direction, considering the variation of the intensity of the evanescent TM an TE modes around the post.

$$
\|\operatorname{grad} I\|_{t}=\frac{I_{T M}-I_{T E}}{\pi / 2(d / 2+r)}
$$

where $I_{T M}$ and $I_{T E}$ are the intensities of the TM and TE evanescent modes respectively. According to the expressions of the coefficients in Eq. 3, one finds that the force in the tangential direction is of the order of $1 \times 10^{-20} \mathrm{~N}$, i.e. 3 orders of magnitude lower than in the radial direction. This is in good agreement with the experimentally estimated force in the tangential direction $F_{t}$. Since a 10images-per-second-acquisition rate camera enables to resolve the dynamics of the trapping in the tangential direction, the resolution of the dynamics in the radial direction would imply using a camera with a $10^{4}$-images-per-secondacquisition rate at least.

We have also noticed a slight displacement of the particles in the upward direction. They are propelled along the guide by the radiation pressure, corresponding to the light propagation in the guide as already observed in other tapered waveguides [27-29]. Since the guide is only $15 \mu \mathrm{m}$ long, this displacement is hardly noticeable.

Multiple particle trapping. So far, we have focused on single particle trapping but several particles could be trapped successively. We may then form particle aggregates with various shapes (see Fig. 5). Even in this case, particles accumulate around the $\theta_{p}$ direction, partly coating the post in that direction. As their number increases they start forming chains or 2 dimensional planar structures as if the particles themselves form patterned surfaces $[30,31]$, that could be used for new functionalities [32]. Dielectric particles could also form pattern structures [33-36]. However, here, the evanescent wave is modified by the presence of already trapped particles, and the incoming new colloids attach themselves to the trapped ones, whereas in the mentioned references, the patterning of particles comes from the evanescent field alone. Besides, the particle aggregation as well as the particle dynamics could be dramatically changed depending on the ellipticity and shape of the trapped particle $[37,38]$. This could indeed leads to new structures with multiple trapped states and particular dynamics.

Conclusion. - As a conclusion, we have experimentally trapped micro-meter size particles in the evanescent wave of a low power laser guided in a $3.5 \mu \mathrm{m}$ diameter multimode waveguide. We find a capture range of $2.25 \mu \mathrm{m}$ from the guide surface. We evidence a migration of the particles around the post towards a specific direction corresponding to the TM direction of the incident light. Such manipulation of micro-objects with low power laser may 


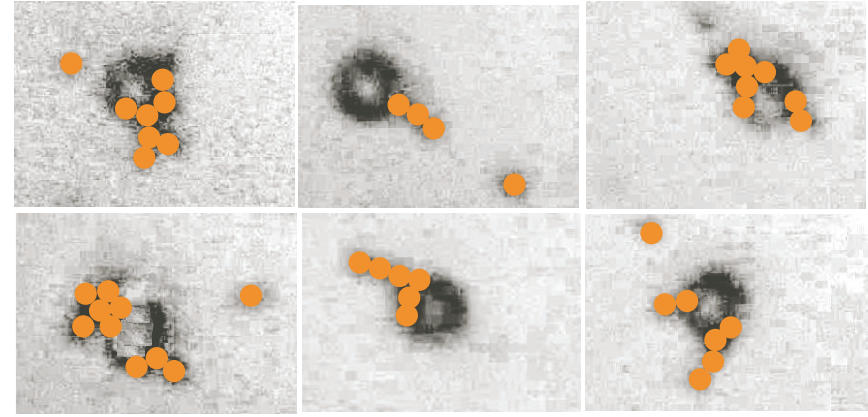

Fig. 5: Pictures showing several particles trapped by the light on the post. The particles have been colored for the sake of clarity.

readily find applications in the handling of very sensitive objects of biological interest, since inherent thermal effects in the vicinity of optofluidics devices that modify or are even detrimental to the objects [39], are here severely reduced.

It could also be a way to control the local stiffness of the interface [40] with light. In porous media, the use of plasmonic patterns, that are known to dramatically enhance the electric fields, leading to high gradients with low power [41], could control or even stop the flow of colloidal particles. This may be of great interest in environment problems, such as filtration or sorting of colloidal pollutants in dedicated microfluidic devices [42]. Our approach can easily be "parallelised" in order to be more efficient, by splitting a high power laser source on thousands of posts.

We would like to thank A. Hubert for early interest, and G. Pécheul for technical assistance.

\section{REFERENCES}

[1] Ashrin A., Dziedzic J. M., BJorkholm J. E. and Chu S., Opt. Lett., 11 (1986) 288.

[2] Ashin A., PNAS, 94 (1997) 4853.

[3] Grier D. G., Nature, 424 (2003) 810.

[4] Moffitt J. R., Chemla Y. R., Smith S. B. and Bustamante C., Ann. Rev. Biochem., 77 (2008) 205.

[5] Righini M., Volpe G., Girard C., Petrov, D. and Quidant R., Phys. Rev. Lett., 100 (2008) 186804.

[6] Grigorenko A. N., Roberts N. W., Dickinson M. R. and Zhang Y., Nat. Photon., 2 (2008) 365.

[7] Juan M. L., Righini M. and Quidant R., Nat. Photon., 5 (2011) 349.

[8] Erickson D., Serey X., Chen Y. F. and Mandal S., Lab Chip, 11 (2011) 995.

[9] Kawata S. and Sugiura T., Opt. Lett., 17 (1992) 772.

[10] GU M., Haumonte J. B., Micheau Y., Chon J. W. M. and Gan X., Appl. Phys. Lett., 84 (2004) 4236.

[11] Gaugiran S., Gétin S., Fedeli J., Colas G., Fuchs A., Chatelain F. and Dérouard J. D., Opt. Express, 13 (2005) 6956.
[12] Grujic K., Helles $\varnothing$ O. G., Hole J. P. and Wilkinson J. S., Opt. Express, 13 (2005) 1.

[13] Hsu L. C., Chen T. C., Yang Y. T., Huang C. Y., Shen D. W., Chen Y. T. and Lee M. C. M., Lab Chip, 13 (2013) 1151.

[14] Maimaiti A., Truong V. G., Sergides M., Gusachenko I. and Chormaic S. N., Sci. Reports, 5 (2015) 9077.

[15] Dullo F. T. and Helles $\varnothing$ O. G., Lab Chip, 15 (2015) 3918.

[16] Squires T. M. and Quake R. S., Rev. Mod. Phys., 77 (2005) 977

[17] Hawkins A. R. and Schmidt H. (Editors), Handbook of optofluidics. (CRC Press, New York) 2010.

[18] Wright S. L., Thompson R. C. and Galloway T. S., Environ. Poll., 178 (2013) 483.

[19] Ether JR. D. S., Pires L. B., Umrath S, Martinez D., Ayala Y., Pontes B., Arajo G. R. De S. , Frases S., Ingold G.-L, Rosa F. S. S., Viana N. B. , Nussenzveig H. M. and Maia Neto P. A., EPL, 112 (2015) 44001.

[20] Kogelnik H., Theory of optical waveguides, edited by TAMiR T. (Springer Berlin Heidelberg) 1988.

[21] Yariv A. and YeH P., Photonics: Optical Electronics in Modern Communications 6th Ed. (Oxford University Press, Oxford) 2006.

[22] Born M. and Wolf E., Principles of optics. 7th Ed. (Cambridge University Press, Cambridge) 1999.

[23] Almans E., and Brevik I., J. Opt. Soc. Am. B, 12 (1995) 2429 .

[24] Volpe G., Quidant R., Badenes G. and Petrov D., Phys. Rev. Lett., 96 (2006) 238101.

[25] Neuman K. C., and Block S. M., Rev. Sci. Instrum., 74 (2004) 2787.

[26] Wada O., and Nakajima M., Electron. Comm. Jpn., 71 (1988) 83

[27] Šiler M., Čižmár T., ŠerÝ, M. and Zemánek P., Appl. Phys. B., 84 (2006) 157.

[28] Schmidt B. S., YAng A. H., Erickson D. and Lipson M., Opt. Express, 15 (2007) 14322.

[29] Brambilla G., Murugan G. S., Wilkinson J. S., and Richardson D. J., Opt. Lett., 32 (2007) 3041.

[30] Lee Y. H., Shi W., Lee H. K., Jiang R.,Phang I. Y., Cui Y., Isa L., Yang Y., Wang J., Ki S. and Ling X. Y., Nat. Commun., 6 (2015) 6990.

[31] Liu Q., Senyuk B., Tang J., Lee T., Qian J., He, S. and Smalyukh I. I., Phys. Rev. Lett., 109 (2012) 088301.

[32] Veiga-Gutiérrez M., Woerdemann M., Prasetyanto E., Denz C. and De Cola L., $A d v$. Mater., 24 (2012) 5199.

33] Mellor C. D., Fennerty T. A., and Bain C. D., Opt. Express, 14 (2006) 10079.

[34] Garcés-Chávez V., Dholakia K., and Spalding G. C., Appl. Phys. Lett., 86 (2005) 031106.

[35] Han X. and Jones P. H., Opt. Lett., 40 (2015) 4042.

[36] Gusachenko I., Truong V. G., Frawley M. C., and Chormaic S. N., Photonics, 2 (2015) 795.

[37] Wilking J. N. and Mason T.G., EPL, 81 (2008) 58005.

[38] Miniretie B. M., Snabre P., Loudet J. C. and Pouligny P., EPL, 100 (2012) 48005.

[39] Serey X., Mandal S., Chen Y. F. and Erickson D., Phys. Rev. Lett., 108 (2012) 048102.

[40] Chang T., Zhang H., Guo Z., Guo X. and Gao H., 
O. Emile et al.

$384 \quad$ Phys. Rev. Lett., 114 (2015) 015504.

385 [41] Valev V., Silhanek A. V., Jeyaram Y., Denkova D., 386 De Clerce B., Petkov V., Zheng X., Volskiy V.,

387 Gillijns W., Vandenbosch G. A. E., Aktsipetrov O.

388 A., Ameloot M., Moshchalkov V. V. and Verbiest

389 T., Phys. Rev. Lett., 106 (2011) 226803.

390 [42] Jokerst J. C., Emory J. M. and Henry C. S., Analyst, 137 (2012) 24 